\title{
Two Theorems on Convergence of Schrödinger Means
}

\section{Per Sjölin ${ }^{1}$}

Received: 4 March 2018 / Published online: 4 October 2018

(c) The Author(s) 2018

\section{Abstract}

Localization and convergence almost everywhere of Schrödinger means are studied.

Keywords Schrödinger equation · Convergence $\cdot$ Localizaton $\cdot$ Sobolev spaces

Mathematics Subject Classification 42B99

\section{Introduction}

For $f \in L^{2}\left(\mathbb{R}^{n}\right), n \geqslant 1$ and $a>1$ we set

$$
\widehat{f}(\xi)=\int_{\mathbb{R}^{n}} e^{-i \xi \cdot x} f(x) d x, \quad \xi \in \mathbb{R}^{n}
$$

and

$$
S_{t} f(x)=\int_{\mathbb{R}^{n}} e^{i \xi \cdot x} e^{i t|\xi|^{a}} \widehat{f}(\xi) d \xi, \quad x \in \mathbb{R}^{n}, \quad t \geqslant 0 .
$$

For $a=2$ and $f$ belonging to the Schwartz class $\mathscr{S}\left(\mathbb{R}^{n}\right)$ we set $u(x, t)=$ $S_{t} f(x) /(2 \pi)^{n}$. It then follows that $u(x, 0)=f(x)$ and $u$ satisfies the Schrödinger equation $i \partial u / \partial t=\Delta u$.

We introduce Sobolev spaces $H_{s}=H_{s}\left(\mathbb{R}^{n}\right)$ by setting

$$
H_{s}=\left\{f \in \mathscr{S}^{\prime} ;\|f\|_{H_{s}}<\infty\right\}, \quad s \in \mathbb{R},
$$

\section{Communicated by Mieczysław Mastyło.}

$凶$ Per Sjölin

persj@kth.se

1 Department of Mathematics, KTH Royal Institute of Technology, 10044 Stockholm, Sweden 
where

$$
\|f\|_{H_{s}}=\left(\int_{\mathbb{R}^{n}}\left(1+|\xi|^{2}\right)^{s}|\widehat{f}(\xi)|^{2} d \xi\right)^{1 / 2} .
$$

In the case $\mathrm{n}=1$ it is well-known (see Sjölin [7] and Vega [9] and in the case $a=2$ Carleson [3] and Dahlberg and Kenig [4]) that

$$
\lim _{t \rightarrow 0}(2 \pi)^{-n} S_{t} f(x)=f(x)
$$

almost everywhere if $f \in H_{1 / 4}$. Also it is known that $H_{1 / 4}$ can not be replaced by $H_{S}$ if $s<1 / 4$.

Assuming $n \geqslant 2$ and $a=2$ Bourgain [1] has proved that (1) holds almost everywhere if $f \in H_{s}$ and $s>1 / 2-1 / 4 n$. On the other hand Bourgain [2] has proved that $s \geqslant n / 2(n+1)$ is necessary for convergence for $a=2$ and all $n \geqslant 2$. In the case $n=2$ and $a=2$, Du, Guth, and $\mathrm{Li}$ [5] proved that the condition $s>1 / 3$ is sufficient. Recently Du and Zhang [6] proved that the condition $s>n / 2(n+1)$ is sufficient for $a=2$ and all $n \geqslant 3$.

In the case $a>1, n=2$, it is known that (1) holds almost everywhere if $f \in H_{1 / 2}$ and in the case $a>1, n \geqslant 3$, convergence has been proved for $f \in H_{s}$ with $s>1 / 2$ (see [7] and [9]).

If $f \in L^{2}\left(\mathbb{R}^{n}\right)$ then $(2 \pi)^{-n} S_{t} f \rightarrow f$ in $L^{2}$ as $t \rightarrow 0$. It follows that there exists a sequence $\left(t_{k}\right)_{1}^{\infty}$ satisfying

$$
1>t_{1}>t_{2}>t_{3}>\cdots>0 \text { and } \lim _{k \rightarrow \infty} t_{k}=0
$$

such that

$$
\lim _{k \rightarrow \infty}(2 \pi)^{-n} S_{t_{k}} f(x)=f(x)
$$

almost everywhere.

We shall here study the problem of deciding for which sequences $\left(t_{k}\right)_{1}^{\infty}$ one has

$$
\lim _{k \rightarrow \infty}(2 \pi)^{-n} S_{t_{k}} f(x)=f(x)
$$

almost everywhere if $f \in H_{S}$. We have the following result.

Theorem 1 Assume $n \geqslant 1$ and $a>1$ and $s>0$. We assume that (2) holds and that $\sum_{k=1}^{\infty} t_{k}^{2 s / a}<\infty$ and $f \in H_{s}\left(\mathbb{R}^{n}\right)$. Then

$$
\lim _{k \rightarrow \infty}(2 \pi)^{-n} S_{t_{k}} f(x)=f(x)
$$

for almost every $x$ in $\mathbb{R}^{n}$. 
Now assume $n=1, a>1$, and $0 \leqslant s<1 / 4$. In Sjölin [8] we studied the problem if there is localization or localization almost everywhere for the above operators $S_{t}$ and the functions $f \in H_{S}$ with compact support, that is, do we have

$$
\lim _{t \rightarrow 0} S_{t} f(x)=0
$$

everywhere or almost everywhere in $\mathbb{R}^{n} \backslash(\operatorname{supp} f)$ ?

It is proved in [8] that there is no localization or localization almost everywhere of this type for $0 \leqslant s<1 / 4$. In fact the following theorem was proved in Sjölin [8].

Theorem A There exist two disjoint compact intervals I and $J$ in $\mathbb{R}$ and a function $f$ which belongs to $H_{s}$ for all $s<1 / 4$, with the properties that (supp $\left.f\right) \subset I$ and for every $x \in J$ one does not have

$$
\lim _{t \rightarrow 0} S_{t} f(x)=0
$$

Let $\omega$ be a continuous and decreasing function on $[0, \infty)$. Assume that $\omega(t) \rightarrow 0$ as $t \rightarrow \infty$. Set

$$
H_{\omega}=\left\{f \in \mathscr{S}^{\prime} ;\|f\|_{H_{\omega}}<\infty\right\}
$$

where

$$
\|f\|_{H_{\omega}}=\left(\int_{\mathbb{R}}|\widehat{f}(\xi)|^{2}\left(1+\xi^{2}\right)^{1 / 4} \omega(|\xi|) d \xi\right)^{1 / 2}
$$

We have the following result.

Theorem 2 The function $f$ in theorem $A$ can be chosen so that $f \in H_{\omega}$.

Theorem 2 shows that the sufficient condition $f \in H_{1 / 4}$ for convergence almost everywhere and localization almost everywhere of Schrödinger means is very sharp. In the case $\mathrm{a}=2$ Theorem 2 was obtained in 2009 (unpublished). After proving Theorem 2 we shall use Theorem 1 to make a remark on the Schrödinger means $S_{t} f(x)$ for the function $f$ which was constructed in [8] to prove Theorem A.

\section{Proofs}

In the proof of Theorem 1 we shall need the following lemma.

Lemma 1 Assume $n \geqslant 1, a>1,0<s<1$, and $0<\delta<1$. Set

$$
m(\xi)=\frac{e^{i \delta|\xi|^{a}}-1}{\left(1+|\xi|^{2}\right)^{s / 2}}, \quad \xi \in \mathbb{R}^{n} .
$$


Then one has

$$
\|m\|_{\infty} \leqslant C \delta^{s / a}
$$

where the constant $C$ does not depend on $\delta$, and $\|m\|_{\infty}$ denotes the norm of $m$ in $L^{\infty}\left(\mathbb{R}^{n}\right)$.

Proof of Lemma 1. We shall write $A \lesssim B$ if there is a constant $C$ such that $A \leqslant C B$. In the case $|\xi| \geqslant \delta^{-1 / a}$ one has

$$
|\xi|^{s} \geqslant \delta^{-s / a} \text { and }|m(\xi)| \lesssim \frac{1}{|\xi|^{s}} \leqslant \delta^{s / a}
$$

Then assume $0 \leqslant|\xi| \leqslant 1$. We obtain

$$
|m(\xi)| \lesssim \delta|\xi|^{a} \leqslant \delta \leqslant \delta^{s / a} .
$$

In the remaining case $1<|\xi|<\delta^{-1 / a}$ one obtains

$$
|m(\xi)| \lesssim \frac{\delta|\xi|^{a}}{|\xi|^{s}}=\delta|\xi|^{a-s} \lesssim \delta \delta^{-(a-s) / a}=\delta \delta^{-1+s / a}=\delta^{s / a}
$$

and the proof of Lemma 1 is complete.

We shall then give the proof of Theorem 1 .

Proof of Theorem 1. We may assume $0<s<1$. We set

$$
h_{k}(x)=(2 \pi)^{-n} S_{t_{k}} f(x)-f(x), \quad x \in \mathbb{R}^{n}, \text { for } k=1,2,3, \ldots
$$

We have $f \in H_{S}$ and we define $g$ by taking

$$
\widehat{g}(\xi)=\widehat{f}(\xi)\left(1+|\xi|^{2}\right)^{s / 2}
$$

It then follows that $g \in L^{2}\left(\mathbb{R}^{n}\right)$.

We have

$$
S_{t_{k}} f(x)=\int e^{i x \cdot \xi} e^{i t_{k}|\xi|^{a}}\left(1+|\xi|^{2}\right)^{-s / 2} \widehat{g}(\xi) d \xi
$$

and

$$
f(x)=(2 \pi)^{-n} \int e^{i x \cdot \xi}\left(1+|\xi|^{2}\right)^{-s / 2} \widehat{g}(\xi) d \xi .
$$


Hence

$$
\begin{aligned}
& h_{k}(x)=(2 \pi)^{-n} \int e^{i x \cdot \xi}\left(e^{i t_{k}|\xi|^{a}}-1\right)\left(1+|\xi|^{2}\right)^{-s / 2} \widehat{g}(\xi) d \xi \\
& =(2 \pi)^{-n} \int e^{i x \cdot \xi} m(\xi) \widehat{g}(\xi) d \xi
\end{aligned}
$$

where

$$
m(\xi)=\left(e^{i t_{k}|\xi|^{a}}-1\right)\left(1+|\xi|^{2}\right)^{-s / 2}
$$

According to Lemma 1 we have $\|m\|_{\infty} \lesssim t_{k}^{s / a}$ and applying the Plancherel theorem we obtain

$$
\left\|h_{k}\right\|_{2}^{2}=c \int|m(\xi) \widehat{g}(\xi)|^{2} d \xi \lesssim\|m\|_{\infty}^{2} \int|\widehat{g}(\xi)|^{2} d \xi \lesssim t_{k}^{2 s / a}\|f\|_{H_{s}}^{2}
$$

It follows that

$$
\sum_{1}^{\infty} \int\left|h_{k}\right|^{2} d x \lesssim\left(\sum_{1}^{\infty} t_{k}^{2 s / a}\right)\|f\|_{H_{s}}^{2}<\infty
$$

and applying the theorem on monotone convergence one also obtains

$$
\int\left(\sum_{1}^{\infty}\left|h_{k}\right|^{2}\right) d x<\infty
$$

We conclude that $\sum_{1}^{\infty}\left|h_{k}\right|^{2}$ is convergent almost everywhere and hence $\lim _{k \rightarrow \infty} h_{k}(x)$ $=0$ and

$$
\lim _{k \rightarrow \infty}(2 \pi)^{-n} S_{t_{k}} f(x)=f(x)
$$

almost everywhere.

Now assume $n=1$ and $a>1$. We set

$$
m(\xi)=e^{i|\xi|^{a}}, \quad \xi \in \mathbb{R}
$$

and let $K$ denote the Fourier transform of $m$ so that $K \in \mathscr{S}^{\prime}(\mathbb{R})$. According to Sjölin [8] p.142, $K \in C^{\infty}(\mathbb{R})$ and there exists a number $\alpha \geqslant 0$ such that

$$
|K(x)| \lesssim 1+|x|^{\alpha} \text { for } x \in \mathbb{R}
$$

For $t>0$ it is then clear that

$$
e^{i t|\xi|^{a}}=m\left(t^{1 / a} \xi\right)
$$


has the Fourier transform

$$
K_{t}(y)=t^{-1 / a} K\left(t^{-1 / a} y\right)
$$

It follows that $S_{t} f(x)=K_{t} \star f(x)$ for $f \in L^{2}\left(\mathbb{R}^{m}\right)$ with compact support. We let $\breve{g}$ denote the inverse Fourier transform of $g$ and choose $g \in \mathscr{S}(\mathbb{R})$ such that supp $\check{g} \subset$ $(-1,1)$ and $\breve{g}(0) \neq 0$. We set

$$
f_{v}(x)=e^{-i x / v^{2}} \breve{g}(x / v), \quad 0<v<1, \quad x \in \mathbb{R} .
$$

According to [7], p.143, one has $\widehat{f_{v}}(\xi)=v g(v \xi+1 / v)$ and

$$
\left\|f_{v}\right\|_{H_{s}} \lesssim v^{1 / 2-2 s} \text { for } 0<s<1 / 4 \text {. }
$$

We shall state three lemmas from [8].

Lemma 2 There exist positive numbers $c_{0}, \delta$ and $v_{0}$ such that

$$
\left|S_{x v^{2 a-2} / a} f_{v}(x)\right| \geqslant c_{0}
$$

for $0<v<v_{0}$ and $0<x<\delta$.

In the remaining part of this paper $\delta$ and $v_{0}$ are given by Lemma 2 . We may also assume that $\delta<1$.

Lemma 3 For $0<v<\min \left(v_{0}, \delta / 4\right), 0<t<1$, and $\delta / 2<x<\delta$ one has

$$
\left|S_{t} f_{v}(x)\right| \lesssim \frac{v}{t^{\gamma}}
$$

where $\gamma=(1+\alpha) / a>0$.

Lemma 4 For $0<v<\min \left(v_{0}, \delta / 4\right), 0<t<1$, and $\delta / 2<x<\delta$ one has

$$
\left|S_{t} f_{v}(x)\right| \lesssim \frac{t}{v^{\beta}}
$$

where $\beta=2 a$.

We shall use these lemmas to prove Theorem 2.

Proof of Theorem 2. Now let $v_{1}$ satisfy $0<v_{1}<\min \left(v_{0}, \delta / 4\right)$ and set $\epsilon_{k}=2^{-k}$, $k=1,2,3, \ldots$

We also set $\mu=\max ((2 a-2) \gamma, \beta /(2 a-2))$ and choose $v_{k}, k=2,3,4, \ldots$, such that $0<v_{k} \leqslant \epsilon_{k} v_{k-1}^{\mu}$ and

$$
\sum_{k=1}^{\infty} \sqrt{\omega\left(1 / v_{k}^{1 / 2}\right)}<\infty
$$


We then set $f=\sum_{k=1}^{\infty} f_{v_{k}}$ and shall prove that $f \in H_{\omega}$.

Arguing as in [8, pp. 145-147], it follows from Lemmas 2, 3, and 4 that with $t_{k}(x)=x v_{k}^{2 a-2} / a$ one has

$$
\left|S_{t_{k}(x)} f(x)\right| \geqslant c_{0}>0
$$

for $\delta / 2<x<\delta$ and $k \geqslant k_{0}$. Hence we do not have $\lim _{t \rightarrow 0} S_{t} f(x)=0$ in the interval $(\delta / 2, \delta)$. Taking $I=\left[-v_{1}, v_{1}\right]$ and $J \subset(\delta / 2, \delta)$ we have supp $f \subset I$ and for every $x \in J$ one does not have $\lim _{t \rightarrow 0} S_{t} f(x)=0$. Thus Theorem 2 follows. It remains to prove that $f \in H_{\omega}$.

We have

$$
\left\|f_{v}\right\|_{H_{\omega}}^{2}=\int\left|\widehat{f_{v}}(\xi)\right|^{2}\left(1+\xi^{2}\right)^{1 / 4} \omega(|\xi|) d \xi \lesssim I_{1}+I_{2}
$$

where

$$
I_{1}=\int_{-1}^{1}\left|\widehat{f_{v}}(\xi)\right|^{2} d \xi \leqslant C v^{2}
$$

and

$$
I_{2}=\int\left|\widehat{f_{v}}(\xi)\right|^{2}|\xi|^{1 / 2} \omega(|\xi|) d \xi
$$

It follows that

$$
\begin{aligned}
I_{2}=\int & v^{2}|g(v \xi+1 / v)|^{2}|\xi|^{1 / 2} \omega(|\xi|) d \xi \\
& =\int v^{1 / 2}|g(\eta+1 / v)|^{2}|\eta|^{1 / 2} \omega\left(\frac{|\eta|}{v}\right) d \eta= \\
& =v^{1 / 2} \int|g(\xi)|^{2}|\xi-1 / v|^{1 / 2} \omega\left(\frac{|\xi-1 / v|}{v}\right) \xi \leqslant C v^{1 / 2} \\
& \times \int_{|\xi-1 / v| \leqslant v^{1 / 2}}|g(\xi)|^{2} v^{1 / 4} d \xi \\
& +C v^{1 / 2} \int_{|\xi-1 / v| \geqslant v^{1 / 2}}|g(\xi)|^{2}\left(|\xi|^{1 / 2}+v^{-1 / 2}\right) \omega\left(v^{-1 / 2}\right) d \xi \\
& \leqslant C v^{3 / 4}+C \omega\left(v^{-1 / 2}\right) .
\end{aligned}
$$

Hence

$$
\left\|f_{v}\right\|_{H_{\omega}}^{2} \lesssim v^{3 / 4}+\omega\left(v^{-1 / 2}\right), \quad 0<v<1
$$


and

$$
\left\|f_{v}\right\|_{H_{\omega}} \lesssim v^{3 / 8}+\sqrt{\omega\left(v^{-1 / 2}\right)}
$$

We have $f=\sum_{1}^{\infty} f_{v_{k}}$ and it follows that

$$
\|f\|_{H_{\omega}} \leqslant \sum_{1}^{\infty}\left\|f_{v_{k}}\right\|_{H_{\omega}} \lesssim \sum_{1}^{\infty} v_{k}^{3 / 8}+\sum_{1}^{\infty} \sqrt{\omega\left(v_{k}^{-1 / 2}\right)}<\infty
$$

since $v_{k} \leqslant \epsilon_{k}$.

We conclude that $f \in H_{\omega}$ and the proof of Theorem 2 is complete.

Remark 1 In Sjölin [8] the function $f$ in Theorem A is given by the formula

$$
f=\sum_{1}^{\infty} f_{v_{k}}
$$

where $v_{k}$ is defined by taking $0<v_{1}<\min \left(v_{0}, \delta / 4\right)$ and $v_{k}=\epsilon_{k} v_{k-1}^{\mu}$ for $k=$ $2,3,4, \ldots$ Here $\epsilon_{k}=2^{-k}$ and $\mu>0$ is given in the proof of Theorem 2. Also let the intervals $I$ and $J$ be defined as in the proof of Theorem 2 . We then set $t_{k}(x)=x v_{k}^{2 a-2} / a$ for $x \in J$ and $k=1,2,3, \ldots$

It is proved in [8] that for every $x_{0} \in J$

$$
\text { one does not have } \lim _{k \rightarrow \infty} S_{t_{k}\left(x_{0}\right)} f\left(x_{0}\right)=0 \text {. }
$$

We now fix $x_{0} \in J$ and shall use Theorem 1 to prove that although (3) holds one also has

$$
\lim _{k \rightarrow \infty} S_{t_{k}\left(x_{0}\right)} f(x)=0 \text { for almost every } x \in J \text {. }
$$

We have $v_{k}<\epsilon_{k}$ and it follows that

$$
0<t_{k}\left(x_{0}\right) \leqslant \epsilon_{k}^{2 a-2}
$$

and

$$
\sum_{1}^{\infty}\left(t_{k}\left(x_{0}\right)\right)^{2 s / a} \leqslant \sum_{1}^{\infty} 2^{-k(2 a-2) 2 s / a}<\infty
$$

for $0<s<1 / 4$. Also $f \in H_{s}$ for $0<s<1 / 4$ and (4) follows from an application of Theorem 1 . 
Remark 2 In the case $a=2$ one has $\mu=2$ and $v_{k}=\epsilon_{k} v_{k-1}^{2}$, and we also have $0<v_{1}<1 / 4$. It can be proved that it follows that

$$
v_{k}=4 \cdot 2^{k-d 2^{k}}
$$

where $d$ is a constant and $d>2$.

Open Access This article is distributed under the terms of the Creative Commons Attribution 4.0 International License (http://creativecommons.org/licenses/by/4.0/), which permits unrestricted use, distribution, and reproduction in any medium, provided you give appropriate credit to the original author(s) and the source, provide a link to the Creative Commons license, and indicate if changes were made.

\section{References}

1. Bourgain, J.: On the Schrödinger maximal function in higher dimensions. Proc. Steklov Inst. Math. 280, 46-60 (2013)

2. Bourgain, J.: A note on the Schrödinger maximal function. J. Anal. Math. 130, 393-396 (2016)

3. Carleson, L.: Some analytical problems related to statistical mechanics. In: Euclidean Harmonic Analysis. Lecture Notes in Mathematics, vol. 779, pp. 5-45. Springer, Berlin (1979)

4. Dahlberg, B.E.J., Kenig, C.E.: A note on the almost everywhere behaviour of solutions to the Schrödinger equation. Harmonic analysis. Lecture Notes in Mathematics, vol. 908, pp. 205-209. Springer, Berlin (1981)

5. Du, X., Guth, L., Li, X.: A sharp Schrödinger maximal estimate in $\mathbb{R}^{2}$. Ann. Math. 186, 607-640 (2017)

6. Du, X., Zhang, R.: Sharp $L^{2}$ estimate of Schrödinger maximal function in higher dimensions, arXiv: $1805.02775 \mathrm{v} 2$

7. Sjölin, P.: Regularity of solutions in the Schrödinger equation. Duke Math. J. 55, 699-715 (1987)

8. Sjölin, P.: Nonlocalization of operators of Schrödinger type. Ann. Acad. Sci. Fenn. Math. 38, 141-147 (2013)

9. Vega, L.: El Multiplicador de Schrödinger, la Funcion Maximal y los Operadores de Restriccion. Departamento de Matematicas. Univ. Autónoma de Madrid, Madrid (1988) 